\title{
The Association of Social Support, Self-Efficacy with the Duration and Complications of Type 2 Diabetes Mellitus (T2DM) in Patients of a Malaysian Primary Care Clinic
}

\author{
Aneesa Abdul Rashid $^{1}$, Zuhra Hamzah², Chai- Eng Tan², Navin Kumar Devaraj ${ }^{1}$ \\ ${ }^{1}$ Department of Family Medicine, Faculty of Medicine and Health Sciences, Universiti Putra Malaysia \\ Serdang, Selangor, Malaysia, ${ }^{2}$ Department of Family Medicine, Faculty of Medicine, National \\ University of Malaysia, Cheras, KL, Malaysia
}

\begin{abstract}
Introduction: $\mathrm{T} 2 \mathrm{DM}$ is a major cause of mortality and morbidity worldwide. Many factors need to be considered when managing patients with T2DM. Social support and self-efficacy are among the components associated with good diabetic self-care, which in turn lead to good diabetic outcomes. These factors are often overlooked but play an important role in patients' management. This study aimed to determine the association of social support and diabetic selfefficacy of T2DM patients with the duration and complications of T2DM in a government primary care clinic
\end{abstract}

Methods: This is a cross sectional questionnaire study, involving 329 patients with T2DM in an urban Malaysian primary care clinic, using the Medical Outcome Study (MOS) Social Support Survey and Diabetic Management Self-Efficacy Scale (DMSES). The scores were analysed with clinical characteristic of the patients (duration and complications of T2DM) using descriptive and inferential statistics.

Results: The mean duration of T2DM were $6.2+4.8$ years, $57.8 \%$ of patients had no complications. Majority of patients had only 1 complication (33.1\%) and 1 patient had 4 complications $(0.3 \%)$. The complications were retinopathy (18.2\%), nephropathy (14.6\%), peripheral neuropathy (12.8\%) and cardiovascular complications (4.3\%). There was a significant correlation for social support with the duration of T2DM $(r=-0.129, p=0.02)$. There were no significant findings for the diabetic self-efficacy scores with duration and complications of T2DM.

Conclusion: We found a significant negative correlation between the duration of T2DM and social support, which suggests that those with a longer duration of diabetes usually requires lesser social support.

Keywords: Social support, self-efficacy, diabetes, primary care 